\title{
Higher order aberrations of the eye: Part one
}

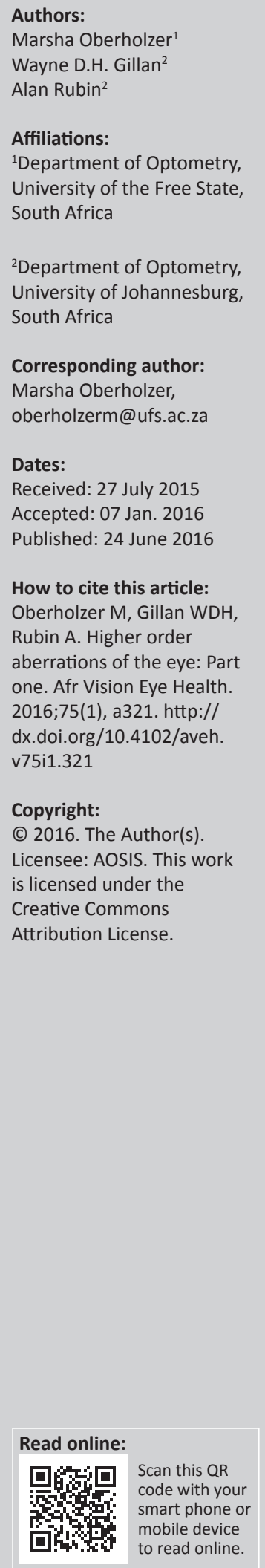

This article is the first in a series of two articles that provide a comprehensive literature review of higher order aberrations (HOAs) of the eye. The present article mainly explains the general principles of such HOAs as well as HOAs of importance, and the measuring apparatus used to measure HOAs of the eye. The second article in the series discusses factors contributing to variable results in measurements of HOAs of the eye.

\section{Introduction}

The present article is the first in a series of two articles that describe the relevant literature concerning higher order aberrations (HOAs) of the eye. The first article largely explains the general and fundamental principles that apply to HOAs as well as indicating exactly which HOAs are of importance, and also the measuring apparatus commonly used to measure HOAs of the eye. The second article (to follow) discusses factors contributing to variable results in measurements of HOAs of an eye that researchers have observed.

\section{Wavefront aberrations in general}

The sensation and perception of vision is created through a complicated process involving optics and neural processing at different levels. The optical or retinal image formed by neural processing may be degraded by blurring resulting from intraocular scatter, diffraction, lower order aberrations (LOAs) that include errors of refraction, and via higher order wavefront aberrations (WAs). 1,2,3,4,5,6 WAs of the eye, and perhaps more specifically HOAs, have been interesting topics in research for more than a decade, and the relevance and impact of such aberrations on the eye's optical quality have been measured and investigated and are of especial importance in the fields of optometry and ophthalmology. ${ }^{7,8}$ For example, WAs have been used to easily and rapidly quantify the optical imaging quality of optical systems such as the eye both before and after refractive surgery. ${ }^{7,8}$ The usefulness and accuracy of Zernike polynomials to facilitate the theoretical understanding of an optical system, based on geometric optical principles, is explained by, for instance, Carvalho ${ }^{9}$ who emphasised that in a so-called ideal or perfect optical system, the perfect wavefront (WF) is considered as a WF that is an imaginary surface formed by the wave tips of rays within the same phase. The perfect optical system is a system without any WAs or scattering of light; however, such a system remains diffraction limited. The propagated rays of this perfect optical system are directed from an object and, should they thereafter pass through a refracting surface such as an optical lens, these refracted rays leave the refracting surface to form a perfect spherical WF with its centre at the image point. ${ }^{9}$

Cerviño et al. ${ }^{5}$ define an aberrated WF as any deviation from the perfect WF that might have been generated from a perfect optical system as described above. The aberrated WF results from heterogeneity within the ocular media and also from local irregularities of optical surfaces. However, aberrations can occur even with centred and supposedly perfect spherical surfaces owing to decentration, tilt or changes in refractive indices within or across surfaces. The visual system is limited by various physical and physiological factors ${ }^{5}$ and heterogeneity of the ocular media is one of the physical factors that degrades retinal image quality. Retinal image quality is also greatly influenced by chromatic and monochromatic WAs, diffraction and scatter. Some authors suggest that these WAs may be the cause of visual distortions such as the degradation of contrast sensitivity and mesopic visual acuity.,3,5 The anatomy of the visual system, including the visual pathway and neurological connections, are some physiological factors that contribute to reduced visual perception and interpretation of the retinal image..$^{5}$ It has been further suggested

Note: This article is based on postgraduate studies by the first author with the supervision of Professors W.D.H. Gillan and A. Rubin of the University of Johannesburg. The degree was conferred on 25 March 2015. 
that haloes, glare and decreased contrast sensitivity may be the result of residual HOAs after refractive surgery on the cornea. ${ }^{4}$

Atchison ${ }^{10}$ points out that WAs include two types or groups of aberrations, namely monochromatic and chromatic aberrations. Chromatic aberrations, like monochromatic aberrations, have a considerable effect on the visual performance of an individual ${ }^{11}$ but, for the purpose of the present article, only monochromatic aberrations of the eye are discussed.

Monochromatic WAs consist of both lower and HOAs. LOAs refer to zero, 1st and 2nd order WAs (which include defocus and astigmatism that relate to sphere, cylinder and axis as used in conventional refractive notation). LOAs can be corrected with normal optical aids such as spectacles or contact lenses but HOAs that involve 3rd and other HOAs cannot easily be corrected with most optical aids as they are the result of irregularities and/or imperfections of the optical system of the eye.,4 Some familiar HOAs are known as spherical aberration (SA) and coma. ${ }^{9}$ As mentioned, both LOAs and HOAs are popularly presented with Zernike polynomials, ${ }^{10}$ and a brief discussion of this and other mathematical approaches follows below.

\section{Zernike polynomial function series}

The WAs of an optical system may be represented by a WA polynomial function named the Taylor series, where the horizontal and vertical co-ordinates of a WA are denoted by $x$ and $y$ values at the entrance pupil or by using the Zernike polynomial function series, which is currently more popular in vision science; but Zernike polynomials are, however, similar to the Taylor series. ${ }^{10,12,13}$ The use of the Zernike polynomial function for representing ocular (or optical) aberrations has been adopted ${ }^{14}$ by the American National Standards Institute ${ }^{14}$ but, for the past 70 years, Zernike polynomials have been used in different scientific fields. Particularly in the last decade or two, Zernike polynomial functions have been used and applied extensively to surfaces such as the human cornea as a standard method of describing WAs. ${ }^{9,14,15}$

Zernike polynomials are a set of orthogonal mathematical basis functions describing the shape of surfaces that are fitted to data. ${ }^{9,13,16,17}$ Each polynomial in this functional series is independent, and this aspect of the Zernike polynomial function makes it unique and encourages its common usage. ${ }^{10,14}$ Zernike polynomials enable decomposition of the complicated and irregular shape of a WA into simpler mathematical components expressed in terms of radial and angular frequencies. ${ }^{15}$ Each function has a single coefficient or value that indicates the amount of the specific WA that, together with other WAs in the series, composes the total WA. ${ }^{16,17}$ These coefficients $\left(C_{n}^{m}\right.$ where the subscripts $n$ and $m$ refer to order and angular frequency respectively) are measured in micrometres or microns $(\mu \mathrm{m})$. These small measurement units ensure that the majority of aberration variance typically found in human eyes are adequately presented. ${ }^{18,19}$ The Zernike polynomial series has several interesting properties and the series has an infinite number of polynomials, usually represented by two real variables, rho $(\rho)$ and theta $(\theta)$. These polynomials are orthogonal to each other in a continuous fashion over the interior of a unit circle. This orthogonal nature of Zernike polynomials indicates that polynomials multiplied by each other across the pupil will, if they are summed, equal zero. This property of Zernike polynomials guarantees that one will be able to construct the data of any continuous surface, provided that sufficient terms are used. Corneas that have not undergone corneal curvature changes as a result of severe physical trauma, keratitis, severe ocular disease or keratoplasty may therefore be satisfactorily presented with Zernike polynomials. ${ }^{910,20}$

Zernike polynomials may be presented in a function pyramid or triangle in which order, denoted by $n$, changes vertically and angular frequency, denoted by $m$, changes horizontally (Figure 1). The polynomials in the Zernike pyramid or triangle may be presented as $-Z_{n}^{m}$ and $+Z_{n}^{m} \cdot{ }^{10,14}$

In Figure $1, Z_{0}^{0}$ in the first row has the order and frequency $n=0$ and $m=0$ and refers to the aberration piston. ${ }^{10,14} Z_{1}^{-1}$ and $Z_{1}^{1}$ where $n=1$ and $m=-1$ or 1 are first order Zernike polynomials. These two polynomials are $y$ - and $x$-tilt or prism and are $90^{\circ}$ rotated functions of each other. ${ }^{10} Z_{2}^{-2}, Z_{2}^{0}$ and $Z_{2}^{2}$ are second order Zernike polynomials where $n=2$ and their corresponding coefficients $\left(C_{2}^{-2}, C_{2}^{0}\right.$ and $\left.C_{2}^{2}\right)$ can be used to determine the refraction or refractive state of the eye. The central term, $Z_{2}^{0}$, represents defocus whereas the two terms for astigmatism are on either side of the central term. The $Z_{2}^{-2}$ polynomial, where $m=-2$, has a maximum value along $45^{\circ}$ and a minimum value along $135^{\circ}$ and is called oblique astigmatism. The other polynomial function, where $m=2,\left(Z_{2}^{2}\right)$, represents against-the-rule astigmatism (positive value) or with-the-rule astigmatism (negative value) and is rotated by $45^{\circ}$ to $Z_{2}^{-2}$ with a maximum value along $180^{\circ}$ and a minimum value of along $90^{\circ} \cdot{ }^{10,14} Z_{3}^{-3}$ (oblique trefoil), $Z_{3}^{-1}$ (vertical coma), $Z_{3}^{1}$ (horizontal coma) and $Z_{3}^{3}$ (horizontal trefoil) are polynomials of the third order where $n=3$. These polynomials present the first order of WAs that are included in the

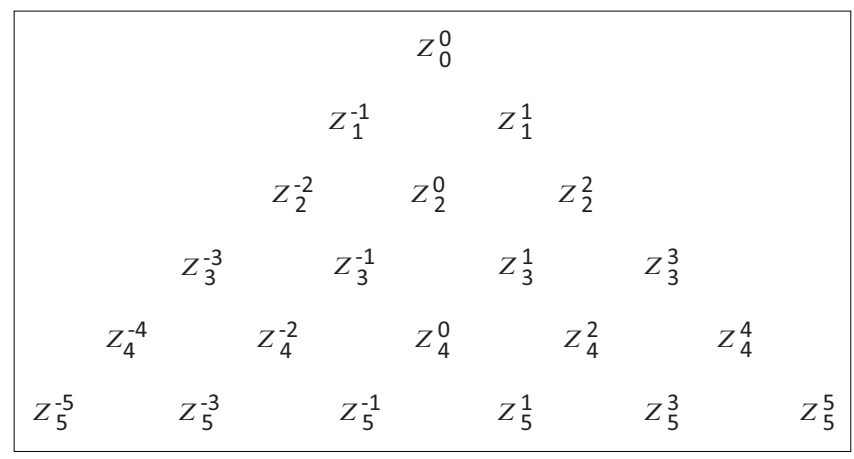

Source: Adapted from Atchison DA. Recent advances in representation of monochromatic aberrations of human eyes. Clin Exp Optom. 2004;87:138-148

The orders continue to infinity but, commonly in vision science, orders beyond 8 are excluded because they have little effect on image quality.

FIGURE 1: The function pyramid presenting the Zernike polynomials from the zero $(n=0)$ to fifth $(n=5)$ order. 
HOAs. ${ }^{10,14}$ From the left, $Z_{4}^{-4}, Z_{4}^{-2}, Z_{4}^{0}, Z_{4}^{2}$ and $Z_{4}^{4}$ are fourth order polynomials $(n=4)$; the centre polynomial $\left(Z_{4}^{0}\right)$ refers to SA and its coefficient $\left(C_{4}^{0}\right)$ may be either positive or negative. The other polynomials from the left are oblique quatrefoil, oblique secondary astigmatism, with or against-the-rule secondary astigmatism and quatrefoil respectively. ${ }^{10,14}$ Progressing further down the Zernike pyramid (not shown in Figure 1), and away from the centre, the pyramid increases in complexity and the modes or terms become even more numerous.

Carvalho et al. ${ }^{21}$ argue that an advantage of Zernike polynomials regarding HOAs is that they describe with high precision some of the conventional Seidel aberrations, such as SAs and coma. Although there is no limit to the number of terms when Zernike polynomials are used (an infinity of HOAs can be represented), a meeting in 2000 of the members of the Vision Science and its Applications Standards task force (VSIA) ${ }^{22}$ discussed the issue of what would be an adequate series of Zernike polynomial functions to present the HOAs of the human eye with sufficient accuracy. The meeting proposed that the first 15 Zernike polynomials and their coefficients (zero to fourth order) was an adequate representation for the most common WAs found in the human eye.

The root-mean-square statistic (RMS) is a measure of the magnitude of the variation of the WAs of a specific eye and can be used to evaluate image quality in human eyes and to compare the WF variation in different eyes. The RMS value is a WA-based optical quality metric and is used to measure the deviation of the aberrated WF from the ideal WF for one or more WAs. Although there are different methods to calculate it, the RMS value is the square root of the sum of the squared individual Zernike coefficients with order $(n)$ and frequency $(m)$ indices of 2 or greater. As all the coefficients are squared, the RMS will always be positive and a RMS value of zero indicates that the optical system is free of WAs.,14,18 The RMS value is a number or scalar; it is probably the most common single metric used to characterise the WAs of an eye. However, if we consider the image degradation on the retina caused by HOAs, the RMS value does not show how a given WA affects visual performance, and is therefore not well correlated with visual performance according to Hunter et al., ${ }^{1}$ Huang, ${ }^{16}$ and He et al. ${ }^{23}$

The ability to measure HOAs of the eye could possibly affect the profession of optometry as we currently know it. Optical limits of the eye imposed by WAs may reduce in the future as a result of refractive surgery, contact lenses and intraocular lenses (IOLs) that may cause improvement of the retinal image quality. Hence, known and more familiar measures of visual performance (e.g. letter acuity) may be less influenced by the optical quality of the retinal image, and visual performance will then only be limited by the neural connections and physiology of both the retina and brain. A new demand for different measures of vision may arise that are more sensitive to measuring even the smallest departures from perfect retinal image quality compared with known measures currently used. ${ }^{24}$

\section{Higher order aberrations of specific importance}

Coma $\left(Z_{3}^{-1}\right.$ and $\left.Z_{3}^{1}\right)$, in contrast to SAs, is represented by asymmetrical Zernike polynomials. Coma also causes asymmetric blurring of an image which, for example, may cause a spot of light to resemble the shape of a comet. Coma mainly occurs when ocular components are not co-axial or from pupil decentration that may increase with pupil dilation and, in terms of HOAs, coma may be the main source of image degradation..$^{25,26}$ Marcos et al. ${ }^{27}$ also suggest that pupil decentration degrades the retinal image quality by introducing coma aberrations. It was previously thought that differences caused by the off-axis position of either the fovea, tilt, misalignment or decentration of the cornea, all with respect to the lens, were the main sources for variations in HOAs, such as with coma. ${ }^{27}$

SA have been of considerable interest to investigators for the last few decades. This WA is rotationally symmetrical ${ }^{10}$ and is represented by a 4 th order Zernike polynomial $\left(Z_{4}^{0}\right)$. SA results from peripheral light rays that are defocused on the retina and causes symmetrical blur of a retinal image. ${ }^{25}$ The coefficient for SA is typically positive for most people in an un-accommodated state and, in instances where SA is large, as with dilated pupils, secondary SA (a 6th order aberration) may also be needed to properly represent SA. With ocular accommodation, $C_{4}^{0}$ (the coefficient of $Z_{4}^{0}$ ) tends to become more negative or less positive in value. ${ }^{10}$

Fang et al..$^{28}$ gave a description of WAs and the optical quality of the eye for a myopic population $(N=113)$ with $6 \mathrm{~mm}$ pupil diameters. They found that the impact of HOAs on retinal image quality may be ranked as follows: $\mathrm{SA}\left(Z_{4}^{0}\right), y$ coma $\left(Z_{3}^{-1}\right)$, $y$ trefoil $\left(Z_{3}^{-3}\right), x$ trefoil $\left(Z_{3}^{3}\right), x$ coma $\left(Z_{3}^{1}\right)$, secondary astigmatism $x\left(Z_{4}^{2}\right)$, secondary astigmatism $y\left(Z_{4}^{-2}\right), x$ tetrafoil $\left(Z_{4}^{4}\right)$, secondary coma $y\left(Z_{5}^{1}\right), y$ tetrafoil $\left(Z_{4}^{4}\right)$, secondary trefoil $y$ $\left(Z_{5}^{-3}\right)$, secondary coma $x\left(Z_{5}^{1}\right), y$ pentafoil $\left(Z_{5}^{-5}\right)$, secondary trefoil $x\left(Z_{5}^{3}\right)$ and $x$ pentafoil $\left(Z_{5}^{5}\right)$. However, the only two aberrations found to be significantly different from zero were SA $\left(Z_{4}^{0}\right)$ and vertical trefoil $\left(Z_{3}^{-3}\right)$, and these two aberrations may therefore be assumed to have the greatest influence on the degradation of a retinal image. Their study further showed that an expected visual benefit may result from reducing five Zernike coefficients in value, namely $C_{4}^{0}$, $C_{3}^{-1}, C_{3}^{-3}, C_{3}^{3}$ and $C_{3}^{1}$. Image degradation may thus be much less when the coefficients of these Zernike polynomials are reduced through, for example, adaptive optics (AO) which is considered in the following section.

\section{Adaptive optics and the measuring apparatus used for the measurement of higher order aberrations}

Visual performance may be determined objectively by various methods including WF aberrometry. WF technology is probably the best option presently for the evaluation of 
HOAs of the eye and is also commonly used in the evaluation of patients before corneal refractive surgery. ${ }^{4}$ The complexity of refractive surgery and also special custom-designed contact lenses require instrumentation that is able to measure the refraction at several points on the cornea. A common problem of machines measuring refraction is variable results owing to dynamic accommodation of the patient as repeated measurements of the same patient are impossible to take with the crystalline lens at exactly the same dioptric power. More modern conventional autorefractors are installed with accommodation devices to relax accommodation, but such devices have their limitations. Conventional instruments are therefore not ideal for evaluating patients for refractive surgery. The need for improved instrumentation was also emphasised by the fact that new laser beams are now used during refractive surgery which have the ability to independently ablate specific parts or spots of the cornea. ${ }^{21}$ Liang et al. ${ }^{29}$ as far as we know, were the first to apply the principles or methods of $\mathrm{AO}$ to the human eye - methods that were initially used to calibrate astronomical telescopes. Astronomical images are degraded owing to WAs that occur during atmospheric turbulence and subtle changes in air temperature. AO was then used to measure and compensate for these environmental WAs. ${ }^{21} \mathrm{AO}$ comprises the optical and mathematical methods that potentially measure and compensate for WAs by means of special optical or aberrometry methods. ${ }^{21,30}$ The importance of $\mathrm{AO}$ in the field of WAs is that AO makes it possible to investigate the influence of HOAs on spatial visual function and to investigate the subjective quality of vision by allowing aberrations to be manipulated. After the correction of HOAs by $\mathrm{AO}$, it is expected that the image quality will improve, resulting in a better and more defined image. $\mathrm{AO}$ is not only reliable in correcting HOAs of the eye but also enables assessment of the visual benefits thereof. ${ }^{31}$

Besides $\mathrm{AO}$, other instruments that are used to measure WAs include aberrometers with a multiple pinhole device developed by Hartmann ${ }^{32}$ and improved by Shack and Platt ${ }^{33}$ in 1971. The Hartmann-Shack (HS) sensor ${ }^{5,9}$ forms the basis for many commercially available aberrometers that are now used in WF analysis. Some critical factors have also been identified that may possibly relate to greater or smaller amounts of HOAs in an eye, as measured with such commercial systems. These critical factors are, amongst others, myopia, $3,15,23,34,35,36,37,38,39,40,41,42,43,44,45,46,47$ pupil diameter, ${ }^{1,7,18,48,49,50,51,52,53,54,55}$ accommodation, $36,47,56,57,58$ age, ${ }^{3,43,44,46,59,60,61,62}$ mydriatic drops, ${ }^{53,63}$ integrity of the tear film ${ }^{64,65,66}$ and mirror symmetry between right and left eyes. ${ }^{13,14,19,37,44,67,68,69,70,71,72,73,74,75}$ Further discussion regarding the variability caused by these factors follows in the second article of the present series.

Instrumentation used to measure WAs may analyse light that is either ingoing or outgoing in nature. The ingoing systems determine WAs by effectively analysing an image onto the retina, whilst outgoing systems place a source of light onto the retina and analyse WAs as light leaves the eye. Earlier methods of determining WAs include subjective methods whereas more modern methods are objective. ${ }^{5}$ Ingoing systems calculate WAs by measuring the altered position of the laser beam projected onto a retina. Several laser rays from the original beam are projected onto the retina and then typically 5-10 frames of the retinal image are captured and defined through a small aperture. These frames may be deformed to a greater or lesser extent but are dependent on the size of the WAs present in the eye examined. The retinal spots are then each compared with their own equivalent ideal reference image, after which the deviation from this reference point is measured and processed and is then representative of the WAs. An example is the Tscherning Principle System that uses a dot pattern mask and allows analysis on a subjective basis; this method has shown good reproducibility for sphero-cylindrical refractions and total RMS Zernike coefficients for HOAs in human eyes. ${ }^{5}$

Outgoing systems use a WF that is divided spatially, such as in the HS method. Aberrometers that use the HS method are essentially fundus cameras that collect reflected light from a single spot of light usually, but not always, with a wavelength of roughly $785 \mathrm{~nm}$, projected onto the retina. As the aberrations of the optics of the eye distort the light, the resulting light emerging from the eye and having passed through the HS micro-lenslets is focused as an array of spots onto a charge-coupled device (CCD). The distance between each of the spots is measured, and its ideal position and these measurements of displacement (in the horizontal and vertical planes) are related to local distortions in the pupil as a result of the optics of the eye and the slope of the emergent WF. The number of micro-lenses in the HS sensor is an important factor because, when the number of micro-lenses (in rows and columns) is doubled, the resolution is multiplied by a factor of four. About 70-75 locations on the pupil are measured via this array of micro-lenses and the WAs are typically represented as a WF-variability map. The advantage of this method is that the sensor has the ability to measure all of the spots in the resulting array simultaneously and thus reduce any variability caused by movement. (Other aberrometers use a scanning laser and collect information sequentially, so head or eye movement may have greater effects.) A limiting factor of this approach, however, is that only a few hundred points can be measured accurately within the pupil, which in turn limits the spatial resolution of the measurement of LOAs. ${ }^{4,21,76,77,78,79}$ However, an aberration map essentially describes the deviation between the measured and reference WFs. Usually, warmer colours in the map represent elevated or phase-advanced portions of the $\mathrm{WF}$, and cooler colours represent the surface depressions or phase retardation for the WF measured. ${ }^{24,80}$ These graphic representations, which include aberration maps of the measured WF and Zernike coefficients, enable one to assess the optical quality of the whole eye and the eye as an optical system $^{64}$; but, complicating matters somewhat, is that Zernike polynomials can also be used to represent single or multiple physical surfaces such as the cornea (i.e. Zernike polynomials can be used to directly model the corneal shape or topography) or instead to represent a WF that only indicates the aberrations of the cornea in isolation from the rest of the eye. The 
measurement of WAs is therefore important in evaluating the eye and its parts as an optical system and may assist in answering many questions such as whether myopes or hyperopes could have reduced visual performance in the absence of ocular pathology, even after being fully corrected with spectacles or contact lenses, and whether in infant or young eyes, this consideration could have an impact on ocular development.

Cerviño et al. ${ }^{5}$ report that previous studies ${ }^{29,81,82}$ have shown good repeatability for measurements taken with HS aberrometers. The HS sensor provides measurements that are regarded as highly accurate and reproducible in the determination of refractive error and comparable to, or even better than that from other autorefractors. The problem of induced instrument myopia in non-cyclopleged eyes, however, must still be considered. ${ }^{5}$ The Zywave aberrometer from Bausch and Lomb makes use of a HS sensor. ${ }^{7}$ This aberrometer is reported by Rodríguez et al. ${ }^{7}$ to improve the signal-to-noise ratio caused by Gaussian noise by a factor of 5 compared with the older Tracey aberrometer and other experimental aberrometers. ${ }^{7}$

For highly distorted corneas, the number of micro-lenses in the rows and columns are important as a too-high resolution may be a disadvantage. In a previous study ${ }^{21}$ by Carvalho et al., computer simulations of HS patterns were implemented for several corneal topographers. Their study showed that, for eyes and corneas with severe curvature changes, as in the case of keratoconus, the HS spots may possibly overlap and therefore the capacity of the software that processes the image and analyses the data, will be reduced.

\section{Conclusion}

Understanding aberrations of the eye and consequent visual degradation involves a complicated collection and application of mathematical formulae. The methods are also vital in properly understanding vision and eye behaviour and development. Although the measurement thereof involves many challenges and both advantages and limitations, understanding both LOAs and HOAs may aid in improving the understanding of many symptoms presented by patients, especially pre- and post-corneal refractive surgery, such as monocular diplopia and vision distortions.

The future development of instruments and methods for measuring and correcting WAs with adaptive optics is currently of great interest, especially amongst refractive surgeons and visual scientists, and we can expect exciting changes in optometry and ophthalmology and the clinical care of many different types of patient.

\section{Acknowledgements Competing interests}

The authors declare that they have no financial or personal relationships which may have inappropriately influenced them in writing this article.

\section{Authors' contributions}

M.O. conducted the literature review as part of her postgraduate studies towards the degree D. Phil (Optometry). W.D.H.G. was the main supervisor during the study and assisted in editorial corrections to the article. A.R. was cosupervisor of the study and also assisted in editorial corrections.

\section{References}

1. Hunter JJ, Campbell MCW, Kisilak ML, Irving EL. Blur on the retina due to higherorder aberrations: Comparison of eye growth models to experimental data. J Vis. 2009;9:1-20.

2. MacRae SM, Applegate RA, Krueger RR. An introduction to wavefront-guided visual correction. In: Krueger RR, Applegate RA, MacRae SM, editors. Wavefront customized visual correction: The quest for Super Vision II. Thorofare: Slack Inc., 2004; p. 3-8.

3. Wei RH, Lim L, Chang WK, Tan DTH. Higher order aberrations in eyes with myopia in a Chinese population. J Refract Surg. 2006;22:695-702.

4. Burakgazi AZ, Tinio B, Bababyan A, Niksarli MD, Asbell P. Higher order aberrations in normal eyes measured with three different aberrometers. J Refract Surg. 2006;22:898-903.

5. Cerviño A, Hosking SL, Montes-Mico R, Bates K. Clinical ocular wavefront analyzers. J Refract Surg. 2007;23:603-616.

6. Ha BJ, Kim SW, Kim SW, Kim EK, Kim T. Pentacam and Orbscan II measurements of posterior corneal elevation before and after photorefractive keratectomy. J Refract Surg. 2009;25:290-295.

7. Rodríguez P, Navarro R, González L, Hernández JL. Accuracy and reproducibility of Zywave, Tracey, and experimental aberrometers. J Refract Surg. 2004;20:810-817.

8. Thibos LN. The 2012 Charles Prentice medal lecture: Wavefront measurement of refractive state. Optom Vis Sci. 2013;90:911-923.

9. Carvalho LA. Accuracy of Zernike polynomials in characterizing optical aberrations and the corneal surface of the eye. Invest Ophthalmol Vis Sci. 2005;46:19151926.

10. Atchison DA. Recent advances in representation of monochromatic aberrations of human eyes. Clin Exp Optom. 2004;87:138-148.

11. Rynders MC, Navarro R, Losada MA. Objective measurement of the off-axis longitudinal chromatic aberration in the human eye. Vision Res. 1998;38: 513-522.

12. Singh S, Ganotra D. Wavefront fitting and comparison of camera aberrations using Zernike circle polynomials. Optik. 2013;124:2379-2386.

13. Porter J, Guirao A, Cox IG, Williams DR. Monochromatic aberrations of the human eye in a large population. J Opt Soc Am A. 2001;18:1793-1803.

14. American National Standards Institute. American National Standard for ophthalmics - methods for reporting optical aberrations of the eye: ANSI Z80.282010. Washington, DC: American National Standards Institute; 2010.

15. Smolek MK. Method for expressing clinical and statistical significance of ocular and corneal wave front error aberrations. Cornea. 2012;31:212-221.

16. Huang D. Physics of customized corneal ablation. In: Krueger RR, Applegate RA MacRae SM, editors. Wavefront customized visual correction: The quest for Super Vision II. Thorofare: Slack Inc., 2004; p. 171-180.

17. Dobos MJ, Twa MD, Bullimore MA. An evaluation of the Bausch \& Lomb Zywave aberrometer. Clin Exp Optom. 2009;92:238-245.

18. Roorda A. A review of basic wavefront optics. In: Krueger RR, Applegate RA, MacRae SM, editors. Wavefront customized visual correction: The quest for Super Vision II. Thorofare: Slack Inc., 2004; p. 9-18.

19. Thibos LN, Hong X, Bradley A, Cheng X. Statistical variation of aberration structure and image quality in a normal population of healthy eyes. J Opt Soc Am. 2002;19:2329-2348.

20. Shannon RR, Wyant JC. Applied optics and optical engineering. Vol. XI. Oxford: Academic Press Inc. (Elsevier); 1992.

21. Carvalho LAV, Castro JC, Carvalho LAV. Measuring higher order optical aberrations of the human eye: Techniques and applications. Braz J Med Biol Res. 2002;35:1395-1406.

22. Thibos LN, Applegate RA, Schwiegerling JT, Webb R, VSIA Standards Taskforce Members. Report from the VSIA taskforce on standards for reporting optical aberrations of the eye. J Refract Surg. 2000;16:5654-5655.

23. He JC, Sun P, Held R, Thorn F, Sun X, Gwiazda JE. Wavefront aberrations in eyes of emmetropic and moderately myopic school children and young adults. Vision Res. 2002;42:1063-1070.

24. Thibos LN, Applegate RA. Assessment of optical quality. In: Krueger RR, Applegate RA, MacRae SM, editors. Wavefront customized visual correction: The quest for Super Vision II. Thorofare: Slack Inc., 2004; p. 55-64.

25. Paquin MP, Hamam H, Simonet P. Objective measurement of optical aberrations in myopic eyes. Optom Vis Sci. 2002;79:285-291.

26. Thibos LN, Ye M, Zang XX, Bradley AB. The chromatic eye: A new reduced-eye model of ocular chromatic aberrations in humans. Appl Optics. 1992;31:3594-3600. 
27. Marcos S, Burns SA, Prieto PM, Navarro R, Baraibar B. Investigating sources of variability of monochromatic and transverse chromatic aberrations across eyes. variability of monochromatic and
Vision Res. 2001;41:3861-3871.

28. Fang L, Wang Y, He X. Optical quality in a myopic population of human eyes. Optik. 2010;121:2003-2007.

29. Liang J, Grimm B, Goelz S, Bille JF. Objective measurement of wave aberrations of the human eye with the use of a Hartmann-Shack wave-front sensor. J Opt Soc Am A. 1994;11:1949-1957.

30. Roorda A, Williams DR. Retinal imaging using adaptive optics. In: Krueger RR Applegate RA, MacRae SM, editors. Wavefront customized visual correction: The quest for Super Vision II. Thorofare: Slack Inc., 2004; p. 43-54.

31. Guo H, Atchison DA, Birt BJ. Changes in through-focus spatial visual performance with adaptive optics correction of monochromatic aberrations. Vision Res. 2008;48:1804-1811

32. Hartmann J. Bemerkungen über den bau und die justierung von spektographen Zeitschrift für Instrumentkunde. 1900;20:47.

33. Shack RV, Platt BC. Production and use of a lenticular Hartmann screen. J Opt Soc Am A. 1971;61:656.

34. Tian Y, Tarrant J, Wildsoet CF. Optical and biometric characteristics of anisomyopia in human adults. Ophthal Physiol Opt. 2011;31:540-549.

35. Karimian F, Feizi S, Doozande A. Higher-order aberrations in myopic eyes. Ophthalmic Vis Res. 2010;5:3-9.

36. Collins MJ, Wildsoet CF, Atchison DA. Monochromatic aberrations and myopia. Vision Res. 1995;35:1157-1163.

37. Buehren T, Collins MJ, Carney LG. Near work induced wavefront aberrations in myopia. Vision Res. 2005;45:1297-1312.

38. Atchison DA, Schmid KL, Pritchard N. Neural and optical limits to visual performance in myopia. Vision Res. 2006;46:3707-3722.

39. Li T, Zhou X, Chen Z, Zhou X, Chu R, Hoffman MR. Relationship between ocula wavefront aberrations and refractive error in Chinese school children. Clin Exp Optom. 2012;95:399-403.

40. Atchison DA. Optical models for human myopic eyes. Vision Res. 2006;46:2236-2250.

41. Gwiazda J, Thorn F, Bauer J, Held R. Emmetropization and the progression of manifest refraction in children followed from infancy to puberty. Clin Vis Sci. 1993;8:337-344.

42. Kwan WC, Yip SP, Yap MK. Monochromatic aberrations of the human eye and myopia. Clin Exp Optom. 2009;92:304-312.

43. Levy Y, Segal O, Avni I, Zadok D. Ocular higher-order aberrations in eyes with supernormal vision. Am J Ophthalmol. 2005;139:225-228.

44. Carkeet A, Luo HD, Tong L, Saw SM, Tan DTH. Refractive error and monochromatic aberrations in Singaporean children. Vision Res. 2002;42:1809-1824.

45. Cheng $X$, Bradley A, Hong X, Thibos LN. Relationship between refractive error and monochromatic aberrations of the eye. Optom Vis Sci. 2003;80:43-49.

46. Hartwig A, Atchison DA. Analysis of higher-order aberrations in a large clinical population. Invest Ophthalmol Vis Sci. 2012;53:7862-7870.

47. Thibos LN, Bradley A, Liu T, Lopez-Gil N. Spherical aberration and the sign of defocus. Optom Vis Sci. 2013;90:1284-1291.

48. Atchison DA, Marcos S, Scott DH. The influence of the Stiles-Crawford peak location on visual performance. Vision Res. 2003;43:659-668.

49. Atchison DA, Scott DH. Contrast sensitivity and the Stiles-Crawford effect. Vision Res. 2002;42:1559-1569.

50. Atchison DA, Scott DH. The Stiles-Crawford effect and subjective measurement of aberrations. Vision Res. 2002;42:1089-1102.

51. Applegate RA, Hilmantel G, Thibos LN. Assessment of visual performance. In: Krueger RR, Applegate RA, MacRae SM, editors. Wavefront customized visual correction: The quest for Super Vision II. Thorofare: Slack Inc., 2004; p. 65-76.

52. Campbell FW, Green DG. Optical and retinal factors affecting visual resolution. J Physiol. 1965;181:576-593.

53. Tabernero J, Atchison DA, Markwell EL. Aberrations and pupil location under corneal topography and Hartmann-Shack illumination conditions. Invest Ophthalmol Vis Sci. 2009;50:1964-1970.

54. Porter J, Yoon G, Lozano D, et al. Aberrations induced in wavefront-guided laser refractive surgery due to shifts between natural and dilated pupil centre locations. J Cat Refract Surg. 2006;32:21-32.
55. Rynders MC, Thibos LN, Bradley A, López-Gil, N. Apodization neutralization: A new technique for investigating the impact of the Stiles-Crawford effect on visual function. Documenta Ophthalmologica Proceedings Series. 1997;60:57-61.

56. López-Gil N, Fernández-Sánchez V, Legras R, Montés-Micó R, Lara F, Nguyen-Khoa $\mathrm{JL}$. Accommodation-related changes in monochromatic aberrations of the human eye as a function of age. Invest Ophthalmol Vis Sci. 2008;49:1736-1743.

57. Atchison DA, Collins MJ, Wildsoet CF, Christensen J, Waterworth MD. Measurement of monochromatic ocular aberrations of human eyes as a function of accommodation by the Howland aberroscope technique. Vision Res. 1995;35:313-323.

58. Awwad ST, Warmerdam D, Lee D, Bowman RW, Cavanagh HD, McCulley JP. Letters to the Editor: Changes in ocular higher order aberrations with accommodation in wavefront-guided LASIK myopic candidates. J Refract Surg. 2009;25:171-172.

59. Jahnke M, Wirbelauer C, Pham DT. Influence of age on optical aberrations of the human eye. Ophthalmologe. 2006;103:596-604.

60. Atchison DA, Markwell EL. Aberrations of emmetropic subjects at different ages. Vis Res. 2008;48:2224-2231.

61. Artal P, Berrio E, Guirao A, Piers P. Contribution of the cornea and internal surfaces to the change of ocular aberrations with age. J Opt Soc Am A. 2002;19:137-143.

62. Au Eong KG, Tay TH, Lim MK. Race, culture and myopia in 110,236 young Singaporean males. Singapore Med J. 1993;34:29-32.

63. Taneri S, Oehler S, Azar DT. Influence of mydriatic eye drops on wavefront sensing with the Zywave aberrometer. J Refract Surg. 2011;27:678-685.

64. Montés-Micó R, Cáliz A, Alió JL. Wavefront analysis of higher order aberrations in dry eye patients. J Refract Surg. 2004;20:243-247.

65. Koh S, Maeda N, Hirohara Y, et al. Serial measurements of higher-order aberrations after blinking in normal subjects. Invest Ophthalmol Vis Sci. 2008;49:133-138.

66. Denoyer A, Rabut G, Baudouin C. Tear film aberration dynamics and vision-related quality of life in patients with dry eye disease. Ophthalmology. 2012;119:1811-1818.

67. Wang L, Koch DD. Ocular higher-order aberrations in individuals screened for refractive surgery. J Cat Refract Surg. 2003;29:1896-1903.

68. Castejón-Mochón JF, López-Gil N, Benito A, Artal P. Ocular wave-front aberration statistics in a normal young population. Vision Res. 2002;42:1611-1617.

69. Marcos S, Burns SA. On the symmetry between eyes of wavefront aberration and cone directionality. Vision Res. 2000;40:2437-2447.

70. Bao J, Le R, Wu J, Shen Y, Lu F, He JC. Higher-order wavefront aberrations for populations of young emmetropes and myopes. J Optom. 2009;2:51-58.

71. Arbelaez MC, Vidal C, Arba-Mosquera S. Bilateral symmetry before and six months after aberration-free correction with the SCHWIND AMARIS TotalTech laser: Clinical outcomes. J Optom. 2010;3:20-28.

72. Armstrong RA. Statistical guidelines for the analysis of data obtained from one or both eyes. Ophthal Physiol Opt. 2013;33:7-14.

73. Murdoch IE, Morris SS, Cousens SN. People and eyes: Statistical approaches in ophthalmology. Br J Ophthalmol. 1998;82:971-973.

74. Karakosta A, Vassilaki M, Plianis S, Elfadl NH, Tsilimbaris M, Moschandreas J. Choice of analytic approach for eye-specific outcomes: One eye or two? Am J Ophthalmol. 2012;153:571-579.

75. Smolek MK, Klyce SD, Sarver EJ. Inattention to nonsuperimposable midline symmetry causes wavefront analysis error. Arch Ophthalmol. 2002;120:439-447.

76. Thibos LN, Hong X. Clinical applications of the Shack-Hartmann Aberrometer Optom Vis Sci. 1999;76:817-825.

77. Ginis HS, Plainis S, Pallikaris A. Variability of wavefront aberration measurements in small pupil sizes using a clinical Shack-Hartmann aberrometer. BMC Ophthalmol. 2004;4:1.

78. Courville CB, Smolek MK, Klyce SD. Contribution of the ocular surface to visual optics. Exp Eye Res. 2004;78:417-425.

79. Basavaraju RM, Akondi V, Weddell SJ, Budihal RP. Myopic aberrations: Simulation based comparison of curvature and Hartmann Shack wavefront sensors. Optics Commun. 2014;312:23-30.

80. Sachdev N, Cairns G, McGhee CNJ. An introduction to wavefront aberrometry and its use in clinical practice [home page on the Internet]. c2003m, p. 32-34 [cited 2012 Jul 12]. Available from: http://www.optometry.co.uk

81. Liang J, Williams DR. Aberrations and retinal image quality of the normal human eye. J Opt Soc Am A Opt Image Sci Vis. 1997;14:2873-2883.

82. Iglesias I, Berrio E, Artal P. Estimate of the ocular wave aberration from pairs of double-pass retinal images. J Opt Soc Am A Opt Image Sci Vis. 1998;15:2466-2476. 